\title{
Chanter la politique : partitions nationales et modulations septentrionales (1789-1799)
}

Singing Politics: National Sheet music and Northern Modulations (1789-1799)

\section{Maxime Kaci}

\section{(2) OpenEdition \\ 1 Journals}

\section{Édition électronique}

URL : https://journals.openedition.org/ahrf/11850

DOI : $10.4000 /$ ahrf. 11850

ISSN : 1952-403X

Éditeur :

Armand Colin, Société des études robespierristes

\section{Édition imprimée}

Date de publication : 1 décembre 2010

Pagination : $79-100$

ISBN : 978-2-200-92634-2

ISSN : 0003-4436

\section{Référence électronique}

Maxime Kaci, «Chanter la politique : partitions nationales et modulations septentrionales

(1789-1799) ", Annales historiques de la Révolution française [En ligne], 362 | octobre-décembre 2010, mis en ligne le 01 décembre 2013, consulté le 23 avril 2022. URL : http://journals.openedition.org/ ahrf/11850 ; DOI : https://doi.org/10.4000/ahrf.11850 


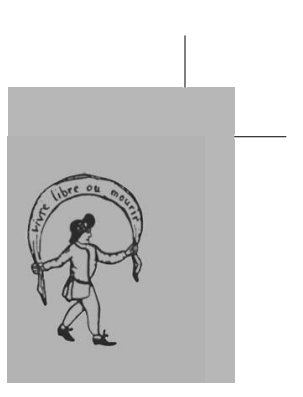

\title{
CHANTER LA POLITIQUE : PARTITIONS NATIONALES ET MODULATIONS SEPTENTRIONALES (1789-1799)
}

\begin{abstract}
Bien que près de 3000 chansons aient été répertoriées pour le seul espace parisien, les œuvres musicales composées durant la période révolutionnaire ont, jusqu'à la dernière décennie $d u x x^{e}$ siècle, suscité peu de travaux. L'analyse combinée des mélodies, des paroles et des contextes d'interprétation, permet de saisir la signification fluctuante des compositions. Les réseaux de circulation qui se tissent dans le Nord attestent d'échanges à double sens avec Paris, et de dynamiques transnationales avérées. Ces dynamiques provoquent des appropriations locales dont témoignent à la fois les parodies et les emplois dans le cadre des engagements collectifs. Les stratégies d'appropriation partagées par des groupes antagonistes relèvent de conflits d'usage intense: chaque parti essaie d'emporter un large soutien en ayant recours aux œuvres à succès.
\end{abstract}

Mots-clés : chanson, circulation, emblème, engagement, Nord.

«Les chanteurs de quai continuent à mettre à contribution les bourses parisiennes [...] le peuple toujours sottement idolâtre et enthousiaste entend brailler d'une voix rauque et aiguë la complainte de Favras, les noms chéris de La Fayette et Bailly : alors il ne peut se dispenser de fouiller dans sa poche et de faire le sacrifice de deux sols pour se procurer la plate rapsodie qui contient les louanges les plus triviales et les plus ridicules des deux plus importants personnages de la capitale ». Les chansons composées et interprétées durant la décennie révolutionnaire suscitent, dès cette époque, des jugements contrastés qui ont orienté, pendant pratiquement deux siècles, les approches historiographiques. Suggérant un 
mépris fondé sur un parti pris esthétique, Louis-Sébastien Mercier, dans son Nouveau Tableau de Paris, exprime une première appréciation : les chansons composées à des fins commerciales visent à flatter de manière triviale les instincts du peuple. Cette attitude de mépris est perceptible tout au long du $\mathrm{XIX}^{\mathrm{e}}$ siècle dans les ouvrages d'historiens nourris par la pensée positiviste et un discours méthodique qui prône l'analyse des sources officielles ou des documents produits par les principaux acteurs de la vie politique. Les propos de Charles Aubertin, professeur de rhétorique et auteur, en 1873, de L'esprit public au XVIII siècle d'après les mémoires et correspondances politiques des contemporains semblent significatifs. Il évoque « ces recueils satiriques, chansons, nouvelles anonymes, rumeurs de la rue et de la place publique que la méchanceté invente ou grossit et que l'oisiveté colporte. Tout ce menu butin de sottisiers du temps, ce résidu fade et cynique des médisances et des perfidies de la vie sociale ne présente à l'observateur, quoi qu'on ait dit, aucun point d'appui assez ferme pour y établir une exacte appréciation des mœurs et de l'esprit d'un siècle $»^{1}$. Pendant plusieurs décennies, les chansons suscitent, avant tout, l'intérêt des folkloristes et des collectionneurs. Constant Pierre, « souschef du secrétariat du Conservatoire National de Musique de Paris » effectue un travail d'érudition précieux en réunissant, dans un catalogue, les références à 2913 chansons composées durant la période révolutionnaire et conservées dans les fonds d'archives parisiens. Sans se départir d'une condescendance esthétique de bon aloi pour l'un des représentants d'une institution prestigieuse créée en 1795, Constant Pierre pressent les apports potentiels d'une étude de ces compositions lorsqu'il affirme :

« Combien de ces chants n'offrent que des idées banales vulgairement exprimées. Certains expriment de manière brutale les sentiments de haine, des passions violentes, ou les pires opinions politiques. Aussi est-ce davantage du point de vue de l'histoire politique et sociale que sous le rapport littéraire, qu'il faut envisager la majeure partie des chansons $»^{2}$.

Dès l'époque révolutionnaire, de nombreux discours insistent, en effet, sur l'utilité politique des œuvres musicales. Un article de la Chronique de Paris publié le 18 mai 1790 indique qu' « il court parmi les rues des chansons à la portée du peuple et faites dans l'esprit de la Révolu-

(1) Charles AuberTin, L'esprit public au XVIII siècle : étude sur les mémoires et correspondances politiques des contemporains de 1715 à 1789, Paris, Didier, 1873.

(2) Constant PIERRE, Les hymnes et chansons de la Révolution, aperçu général et catalogue avec notices historiques, analytiques et bibliographiques, Paris, Imprimerie nationale, 1904, p. 31. 


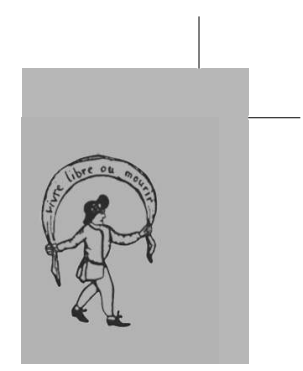

tion [...] nous avons vu avec plaisir ce genre d'amusement si souvent employé par l'ancienne police à distraire le peuple, à l'égarer, ramené à un destin louable contre les menées de la superstition et du fanatisme $»^{3}$. L'usage des chansons à des fins pédagogiques face à la superstition soulève, d'emblée, un questionnement sur l'intérêt des acteurs politiques du moment révolutionnaire à faire circuler des discours en musique. L'évocation de l'esprit de la Révolution semble indiquer, que loin d'être des productions anecdotiques, ces œuvres exprimeraient un ensemble de valeurs et de symboles partagés. Toutefois ce constat ne résout pas le problème de la démarche historique à adopter. Comme l'a souligné Yves Borowice, l'analyse de ces compositions musicales demeure souvent cantonnée à une présentation des «chansons miroirs » assimilées rapidement aux reflets d'une époque, à la traduction dans l'ordre symbolique des configurations connues du contexte sociopolitique ${ }^{4}$. Cette approche, sans être dépourvue d'intérêt, demeure particulièrement appauvrissante puisque la chanson est alors moins considérée comme une source à valeur heuristique que comme l'aimable illustration de dynamiques qui la dépassent. Au moment du Bicentenaire de la Révolution, plusieurs ouvrages composés dans le but de diffuser auprès du plus grand nombre le patrimoine musical révolutionnaire, se sont caractérisés par le recours essentiellement illustratif à la chanson ${ }^{5}$. Pourtant, les historiens des mentalités révolutionnaires ont attiré l'attention sur les fonctions spécifiques des supports musicaux. Georges Lefebvre, dès 1934, dans son article sur « les Foules révolutionnaires », affirmait que les imprimés, les discours et les chansons pouvaient contribuer «à former la mentalité collective $»^{6}$. Michel Vovelle, plus récemment, dans sa préface à l'ouvrage de Robert Brécy, les considère comme un enjeu pour capter les faveurs de l'opinion ${ }^{7}$.

Bien que quelques réflexions ponctuelles aient été publiées avant le Bicentenaire ${ }^{8}$, il fallut attendre la dernière décennie $\mathrm{du} \mathrm{xx}^{\mathrm{e}}$ siècle pour que s'esquisse véritablement une approche renouvelée des chansons

(3) Chronique de Paris, 18 mai 1790, cité par Constant Pierre, op. cit., p. 2.

(4) Yves BorowicE, « La trompeuse légèreté des chansons. De l'exploitation d'une source historique en jachère : l'exemple des années trente », Genèses, n ${ }^{0}$ 65, décembre 2005, p. 98-117.

(5) Robert Brécy, La Révolution en chantant, Paris, éd. Van de Velde, 1988; Georges MARTY Dictionnaire des chansons de la Révolution, Paris, Tallandier, 1988.

(6) Georges LefeBvre, «Les foules révolutionnaires », 1934, rééd. dans La Grande Peur de 1789; suivi de : Les foules révolutionnaires, Paris, Armand Colin, 1988

(7) Robert BRÉCY, op. cit.

(8) Cornwell B. Rogers, «Songs-colorful propaganda of the French Revolution », The Public Opinion Quarterly, vol. 11, $n^{\circ}$ 3, 1947, p. 436 à 444; James A. Leıth, « Music as an ideological weapon in the French Revolution », Historical Papers, vol. 1, nº 1, 1966, p. 126 à 140. 
composées durant le XVIII ${ }^{\mathrm{e}}$ siècle. Claude Grasland a ainsi proposé une méthode quantitative afin de dégager les caractéristiques formelles des chansons produites durant la Régence ${ }^{9}$. Dans son ouvrage Singing the French Revolution, Laura Mason a, quant à elle, porté un éclairage nouveau sur les œuvres composées durant la période révolutionnaire en effectuant une analyse détaillée de leur contenu et de leurs usages ${ }^{10}$. Toutefois, l'absence d'une présentation de la méthode d'analyse soulève plusieurs questions puisque l'historienne ne s'attarde pas à justifier ses choix. At-elle analysé l'ensemble des quelque trois mille œuvres répertoriées par Constant Pierre? Les extraits étudiés en détail correspondent-ils à des discours en musique et à des usages jugés représentatifs? Enfin, les deux approches ont en commun d'être centrées sur l'espace parisien. Or la circulation avérée à l'échelle du royaume, puis du territoire national, des productions musicales pose avec acuité la question de leur visée informative et de leur rôle dans la construction des événements. La problématique de la circulation et des effets induits par les interprétations orales entre en résonance avec des travaux récents tels que ceux de François Ploux qui, à propos de la circulation des rumeurs au $\mathrm{XIX}^{\mathrm{e}}$ siècle, souligne la distorsion qu'implique la transmission de bouche à oreille ${ }^{11}$. En tenant compte, d'une part, des premiers acquis historiographiques nés de l'analyse renouvelée des chansons et, d'autre part, des questionnements récents sur les modalités de circulation, de nouvelles perspectives de recherche s'ouvrent pour l'étude conjointe d'un support et d'une pratique.

\section{Paroles, mélodies et mise en contexte : les trois jalons d'une démarche historique}

Les chansons, définies comme des associations de paroles versifiées ou rythmées selon les règles de la prosodie, et dotées d'une mélodie plus ou moins appropriée, constituent une source au statut mixte, entre oralité et écrit. Les mélodies, comme moyen mnémotechnique, constituent un élément fondamental dans le processus de diffusion des œuvres puisqu'elles contribuent à les rendre à la fois attrayantes et facilement assimilables. Pierre Jean-Baptiste Nougaret, écrivain, parolier, auteur en l'an II d'un recueil de "Chansons de guerre pour les soldats français », témoigne

(9) Claude Grasland, «Chansons et vie politique à Paris sous la Régence », Revue d'Histoire Moderne et Contemporaine, $\mathrm{n}^{\circ}$ 37, 1990, p. 537-570.

(10) Laura MAson, Singing the French Revolution, Cornell University Press, 1996.

(11) François Ploux, De Bouche à oreille, Paris, Flammarion, 2003. 
d'une connaissance précise des enjeux que recouvre le choix d'une mélodie lorsqu'il affirme : «Afin que mes hymnes soient plus populaires, plus utiles, je ne souhaite pas seulement que les compositeurs les embellissent avec des accords harmonieux, mais bien plus qu'ils les intègrent sur des airs connus $\rangle^{12}$. Durant la décennie révolutionnaire, d'après le catalogue de Constant Pierre, 93,5\% des œuvres sont ainsi écrites sur des timbres, c'est-à-dire sur des structures musicales connues du grand public. En d'autres termes, seules 6,5\% des chansons sont composées sur des airs originaux. Par conséquent, l'analyse des discours en musique ne peut se réduire ni aux procédures diverses et connues du commentaire de texte, ni aux approches sémiologiques. Deux facteurs doivent notamment être pris en considération. La structure musicale, jamais neutre, et le contexte d'interprétation, qui relève à la fois des acceptions attribuées à l'œuvre et de ses modalités d'utilisation, contribuent à forger des significations particulières et évolutives. Ces significations procèdent, tout d'abord, de la relation qui se tisse entre une musique populaire dont le texte originel est connu de tous, et des paroles nouvelles adaptées au contexte. Ce jeu intertextuel, fondé sur la réutilisation d'une même mélodie, constitue un puissant ressort satirique. Pour ne citer que deux exemples : la veuve Ferrand compose, en 1790, une chanson intitulée « La chute de la noblesse » sur l'air « De haut en bas »; dans «O filii national», un auteur anonyme dénonce les pratiques du haut clergé en parodiant l'air du célèbre cantique «O filii et filiae ».

Dans un premier temps, une démarche quantitative permet de préciser ces significations particulières qui dépendent à la fois des paroles, de la mélodie et du contexte d'interprétation. L'analyse, qui n'a pas encore été menée à son terme, des thématiques et des procédés littéraires et musicaux utilisés pour transmettre des opinions politiques vise à saisir les rythmes de la production, les thèmes récurrents et, pour ainsi dire, les phénomènes de mode ${ }^{13}$. Si ces données chiffrées ne peuvent constituer une fin en soi, elles permettent de remettre en cause certaines idées reçues. Ainsi, la production de discours en musique n'est pas la caractéristique exclusive des patriotes avancés. Entre 1789 et 1792, par la diffusion intense de périodiques satiriques dans lesquels les chansons tiennent une place importante, Press, 1989

(12) Cité par Emmet Kennedy, A cultural History of the French Revolution, Yale University

(13) Une première grille d'analyse a été élaborée dans le cadre d'un mémoire de Master, M. KACI, Les partitions politiques à travers les chansons durant la Révolution française (1787-1799), Lille 3, Master 1, 2005. 
les défenseurs des prérogatives royales et des hiérarchies traditionnelles introduisent dans le débat politique de nouvelles figures de l'engagement révolutionnaire. François Marchant, dans ses Sabbats Jacobites, met ainsi en scène, dès le 27 avril 1791, la figure du sans-culotte dans une chanson intitulée «La Ronde des sans-culottes $\rangle^{14}$. L'emploi initial du terme doté d'une lourde charge péjorative est, à cette date, bien loin du modèle analysé en détail, pour les années ultérieures, par Haïm Burstin ${ }^{15}$.

Une des tentations premières, pour qui souhaite regrouper des données sur les chansons révolutionnaires, consiste à évaluer leur degré de politisation à l'aune de leurs thématiques. Or toute chanson peut être considérée comme potentiellement politique dès lors que l'on admet que la signification de l'œuvre est indissociable du contexte d'interprétation. Plutôt que de chercher à distinguer chansons politiques et apolitiques à partir des paroles, l'évaluation chiffrée du nombre de chansons comportant un récit des faits mettant en jeu le devenir de la Révolution permet de préciser leur rôle dans la construction de l'événement. Selon Bruno Brévan, ce serait l'événement qui susciterait la chanson durant la période révolutionnaire ${ }^{16}$. Pour l'année 1793, qui s'avère l'une des plus prolixes de la décennie, le corpus établi par Constant Pierre révèle la prépondérance des chansons se rapportant à la guerre dont une soixantaine évoque la prise de Toulon. 50 chansons concernent les assassinats de Marat et de Lepeletier; la fête de la Fédération et l'exécution de Louis XVI totalisent 30 œuvres chacune; la fête de l'Unité et de l'Indivisibilité de la République du 10 août 1793 et l'adoption du nouvel acte constitutionnel font l'objet de 35 chansons. Au total, pour l'année 1793, sur les 590 chansons répertoriées par Constant Pierre, plus de 300 portent sur les principaux événements nationaux. L'importance de la chanson comme support d'information semble dès lors confirmée.

Toutefois, l'analyse chiffrée d'un corpus local, provenant de la France septentrionale, vient nuancer ce premier constat en révélant les biais du corpus parisien et l'importance des motivations diverses qui soustendent la réception des œuvres. Un anonyme réalise, entre 1788 et 1793 , un recueil manuscrit comprenant 446 chansons qu'il semble considérer, au regard du contexte et de ses goûts personnels, comme dignes d'être trans-

(14) Annie Gefrroy, «Sans-culotte, novembre 1790-juin 1792 », dans Annie GefFroy, Jacques Guilhaumou, Sylvia Moreno (dir.), Dictionnaire des usages socio-politiques, fasc. 1 « Désignants sociopolitiques », Paris, Klincksieck, 1985, p. 159-186.

(15) Haïm Burstin, L'invention du sans-culotte, Paris, O. Jacob, 2005. PUF, 1980

(16) Bruno Brévan, Les changements de la vie musicale parisienne de 1774 à 1799, Paris, 
crites $^{17}$. Dans cet ensemble, la plupart des chansons sont des romances, des couplets sur les amours pastorales, ou des descriptions à tonalité grivoise. Sur les 446 chansons recopiées, 34 seulement comprennent des références explicites ou symboliques au contexte révolutionnaire. Le premier événement politique mentionné dans les «Couplets à la Nation» est la tenue des États généraux de 1789. Cette composition est suivie de 14 chansons qui évoquent aussi bien les grandes cérémonies parisiennes comme la fête de la Fédération, que les événements caractéristiques de la vie locale comme les succès militaires à travers notamment « Une chanson des citoyennes de Dunkerque sur l'attaque contre Dunkerque du 22 août au 8 septembre $1793 »$. Au total sept chansons portent sur des événements locaux, huit sur des événements nationaux. En se gardant de toute généralisation abusive à partir d'un recueil partiel et partial qui n'engage que son auteur, plusieurs enseignements peuvent, tout de même, être dégagés. Tout d'abord, les œuvres, parce qu'elles conservent un caractère ludique inhérent, ne soulèvent pas mécaniquement l'enthousiasme par le simple fait qu'elles recèlent des références au contexte sociopolitique. Les événements révolutionnaires ont encouragé une production accrue, un renouvellement des thèmes mais les pratiques et les goûts hérités, comme le suggère l'attrait pour les romances, n'ont pas complètement disparus. Le deuxième enseignement réside dans la nécessité d'articuler l'analyse de l'œuvre à celle des pratiques, ici celle des choix de transcription par un individu, pour évaluer l'impact des chansons à travers les modalités de réception. Ces modalités sont dépendantes de dynamiques de circulation complexes entre Paris et la Province. En circulant, les significations des œuvres sont souvent détournées, ce qui explique la difficulté des acteurs politiques à maîtriser cette « arme révolutionnaire » qui sévit également hors de la capitale comme l'a souligné Frédéric Derne ${ }^{18}$.

\section{Dynamiques nationales et transnationales : la circulation des chansons comme enjeu politique}

Le succès des principales œuvres procède en pratique de dynamiques de circulation complexes, et dépendantes de la diversité des contextes locaux. Michel Espagne, par son travail sur les transferts culturels, a remis en cause la notion d'influence qui tend à rabattre la dyna-

(17) AD Pas-de-Calais, collection Barbier, 4J6.

(18) Frédéric DeRne, «La chanson, arme révolutionnaire et chambre d'écho de la société en Auvergne », Annales historiques de la Révolution française, $\mathrm{n}^{\circ}$ 341, 2005, p. 25-51. 
mique de l'échange du récepteur sur le producteur, en supposant une relation immédiate, quasi magique. Pour lui, «c'est la conjoncture et le contexte d'accueil qui définit largement ce qui peut être importé $[. .$.$] ou$ ce qui doit être réactivé pour servir les débats de l'heure $\gg^{19}$. L'étude de la circulation et de la réception locale des chansons dans la France septentrionale témoigne ainsi de dynamiques multiples. Si le foyer émetteur parisien joue un rôle important de pourvoyeur en compositions nouvelles, il ne constitue pas un centre d'impulsion unique. Des circulations à double sens peuvent être identifiées. Du point de vue thématique, les événements provinciaux sont eux aussi chantés à Paris et contribuent à façonner auprès des Parisiens une représentation des espaces périphériques et de leurs engagements. Les Actes des Apôtres publient ainsi, dans le chapitre 248, « la Complainte sur les excès commis à Douay les 16 et 17 mars 1791 ». Cette chanson fait référence à l'émeute frumentaire dite des Goulottes, du nom des tuyaux de bois utilisés pour charger les bateaux en grains. Le 16 mars, le négociant Nicolon, qui avait protesté contre la destruction par la population de ces tuyaux, est malmené par la foule et finalement massacré tout comme l'imprimeur Derbaix qui avait essayé de lui porter secours. La fureur des émeutiers est dénoncée par un jeu parodique fondé sur l'association d'une description des violences et le choix d'un timbre au titre évocateur, « De la raison nous abjurons l'empire ».

« Complainte sur les excès patriotiques commis à Douay, les 16 et 17 mars $1791 »^{20}$

Air : De la raison nous abjurons l'empire

Nous avons vu le Savoyard atroce,

Pour détrousser sûrement un passant

Lâcher son ours; et cet acte féroce

Dans ses canaux a glacé notre sang

Ah! Frémissons la horde scélérate

De nos Jacots déchaînant d'autres ours

Contre le clerc, contre l'aristocrate

De ses horreurs poursuit l'infâme cours;

(19) Michel Espagne, Les transferts culturels, Paris, PUF, 1999.

(20) Actes des Apôtres, vol. 9, n 248, p. 13. 


\section{$[\ldots]$}

Vous l'éprouvez, douaisiens peu sages;

Les malheureux Derbaix et Nicolon

Assassinés par des anthropophages

Chez nos neveux vont flétrir votre nom

Jusqu'à présent d'un club abominable

Vous aviez su repousser les leçons

Des Desmoulins la doctrine exécrable

Vous aurait-elle inspiré ces poisons?

Paris a vu des monstres en furie

Se disputer les membres de Foulon

Mais vous, poussant plus loin la barbarie,

Avez deux fois fait mourir Nicolon

Non le sommeil, ce baume salutaire

Qui rend la paix à nos sens agités

De la fureur d'un peuple sanguinaire

N'a pu calmer les bouillons irrités

Qui punit-on de tant et tant de crimes

C'est l'innocent, et nos nouveaux Linards

Encor fumans du sang de leurs victimes

Pour d'autres coups aiguisent leurs poignards

Ah! loin de nous un régime sauvage

Qui voit le meurtre et ne l'empêche pas,

N'a que des fers pour les bons qu'il outrage,

Et de faveurs que pour les scélérats.

Les références précises aux événements douaisiens sont, dans le but de sensibiliser un public large, intégrées à un jeu de comparaison entre diverses émeutes, notamment parisiennes à travers l'évocation de Foulon. La chanson progresse alors par élargissement, de la présentation des victimes douaisiennes à la dénonciation d'un club puis d'un régime. L'œuvre musicale permet donc l'instrumentalisation d'un récit d'émeute au profit d'un discours proprement politique visant à orienter les opinions du plus grand nombre. 
L'analyse des circulations spatiales indique que les productions provinciales sont diffusées dans la capitale. Pour la France septentrionale, ce sont, avant tout, les péripéties militaires qui sont l'objet de mises en chanson. D'après le catalogue de Constant Pierre, 12 chansons évoquent explicitement le siège de Lille par les armées autrichiennes en 1792. Dans cet ensemble d'œuvres, deux sont, à coup sûr, des productions locales. "L'hymne aux lillois » est composé peu après le recul des troupes autrichiennes, par un garde national de Douai sur «l'air des Marseillais ». Le choix de l'air illustre clairement l'articulation établie entre la symbolique nationale et les références aux événements locaux. Cette chanson semble circuler à Paris sous forme de feuilles volantes. La seconde, intitulée «Chanson lilloise au sujet du bombardement de Lille » insiste sur la débâcle des Autrichiens. Elle est écrite sur un air qui prend une forte coloration ironique, « Jadis un empereur célèbre ». Elle est l'œuvre d'un négociant lillois dénommé Gouchon, qui n'est pas un novice dans le domaine des écrits politiques puisque, dès 1790, il publie dans le journal lillois L'abeille patriote, une fable satirique intitulée « L'abeille et le frelon ». Sa chanson est publiée dans un autre périodique local, la Gazette $d u$ département $d u$ Nord, le 27 octobre 1792, et est diffusée à Paris sur ce support.

Enfin, les indices de circulations transfrontalières remettent en cause les affirmations de contemporains français tels que le parolier Roussel qui écrit dans un article publié dans la Chronique de Paris en octobre 1792 : «L'Italie est le pays du chant, la France est le pays des chansons ». La pratique de la chanson et son usage répété dans un contexte de tension politique sont certes répandus dans la France révolutionnaire, mais ne constituent pas une spécificité française. En Angleterre, à la même période, alors que s'accuse l'opposition entre partisans des réformes et loyalistes, une lettre signée Fidelia recèle une proposition significative adressée aux membres de l'Association for preserving Liberty and Property:

« Ils [les membres des classes populaires] sont incapables de lire ou de comprendre un discours sérieux et bien écrit destiné à les mettre dans le droit chemin, mais par le biais des chansons populaires, il est certain que l'instruction peut être efficacement transmise, et l'esprit patriotique attisé; en témoigne le "Ça Ira" : chaque domestique et sa femme, chaque fille du pays et son fiancé, dans les villes comme les villages, achèteront une chanson à un demi penny composée sur un air à succès évoquant ces idées. J'en ai écrit une intégralement conçue pour que ces gens puissent la 
comprendre. Si vous l'approuvez, j'en écrirai bien d'autres, que je vous enverrai pour de nombreuses raisons $»^{21}$.

Cette suggestion ne reste pas sans suite puisque, dès janvier 1793 , alors que les tensions entre la France et l'Angleterre s'accentuent, l'Association for preserving Liberty and Property distribue environ 300 chansons, par l'intermédiaire des agents du "victualling office 》 (bureaux chargés de l'approvisionnement au sein de l'administration de la Royal Navy), aux marins présents dans les ports de Plymouth et Portsmouth ${ }^{22}$.

Les Belges réfugiés en France septentrionale depuis la tentative d'indépendance des États Belgique Unis s'impliquent également dans ce processus de circulation et de références croisées. Les réfugiés vonckistes ont, eux aussi, recours aux discours en musique. «Une quantité innombrable de chansons » aurait été diffusée de part et d'autre de la frontière entre Valenciennes et Mons, selon deux individus qui réclament leur dû au sous-comité vonckiste de Valenciennes pour avoir accepté de prendre en charge cette distribution ${ }^{23}$. Ces deux exemples anglais et belges illustrent à la fois la mise en place de dynamiques de circulation transnationales et, dans un même temps, la difficulté de saisir la nature précise de ces échanges puisque aucune transcription des œuvres distribuées n'a, pour l'heure, été retrouvée. La constitution d'un corpus permettant de préciser les modalités et les contextes de réception rend alors possible l'évaluation des effets de ces circulations sur la population locale et sur les prises de position collectives.

\section{Les interprétations collectives dans le Nord ou les logiques d'une appropriation}

L'étude des interprétations collectives, dans le département du Nord, permet de préciser les voies par lesquelles les chansons deviennent des emblèmes d'engagement aux significations fluctuantes. Dans Marianne au combat, Maurice Agulhon a clairement distingué les symboles qui néces-

(21) British Library, Additional Manuscripts, $\mathrm{n}^{\circ}$ 16920, folio 99, lettre de Fidelia du 4 décembre 1792 : «they [the lower class of the people] are incapable of reading or understanding any good or serious adress to set them right, but through the medium of vulgar ballad, surely much instruction can be conveyed, and much patriotic spirit awakend; witness Ca Ira, every serving man, and maid, every country girl and her sweetheart, in towns and villages will buy anhalfpenny ballad to a popular tune upon this idea. I have written one calculated entirely for the medium of their understandings. If you approve it, I will write many others, I send it to you for many reasons ».

(22) British Library, Additional Manuscripts, $n^{\circ}$ 16924, folio 108, lettre de George Cherry membre du victualling office à Moore secrétaire de l'association le 19 janvier 1793 .

(23) Archives générales du Royaume, fonds États Belgique Unis : nº 218/3, folio 89. 
sitent une culture particulière pour être compris, des emblèmes dont la signification, fruit d'une accoutumance, serait perçue par tous. L'interprétation des chansons peut donc être assimilée à une pratique emblématique puisqu'en participant à cette accoutumance, elle confère aux ouvres une signification renouvelée dans un contexte spécifique. Ce constat appelle l'étude non seulement de l'offre musicale qui circule dans la région, mais aussi de la demande d'un public divers qui ne saurait être figé dans une attitude passive, comme nous l'avons déjà suggéré. Parce que la chanson est un bien commercialisé, le succès des œuvres relève d'attitudes de consommation connues : le public achète après avoir effectué un choix qui peut aussi bien révéler des goûts personnels que des orientations politiques assumées. Ce choix implique une sélection, des recherches et parfois des déplacements. Ainsi, Marie-Anne Duprey, âgée de 67 ans, « ci-devant tisseuse » et mère d'un émigré, n'hésite pas le 6 germinal an III à se rendre à Tournai après avoir été informée que l'on y vendait des almanachs de l' " ancienne forme ». Elle qui aurait déclaré «aimer les Lillois et les Tournaisiens parce qu'ils sont contre-révolutionnaires », s'emporte lorsqu'on lui propose d'acheter le Chanteur républicain, et précise «n'avoir que f...de ces sacrés almanachs de carmagnoles $»^{24}$. Par ailleurs, le public consommateur devient souvent interprète et, du même coup, agent d'une appropriation qui confère une signification nouvelle à l'œuvre dans un contexte particulier. Ces considérations ayant trait à la demande et aux modalités de réception de l'œuvre soulèvent alors le problème des sources disponibles. Les documents produits par les autorités locales s'avèrent précieux, mais profondément orientés par un usage utilitaire des références aux chansons. Celles-ci sont considérées, avant tout, comme un baromètre de l' « esprit public ». Par conséquent un déséquilibre se manifeste, entre d'une part, des chansons jugées séditieuses qui font l'objet d'un développement minutieux comportant une description du contexte d'interprétation, et parfois une transcription des paroles et de la musique, et d'autre part, des interprétations considérées comme patriotiques, donc satisfaisantes, qui ne nécessitent pas, aux yeux des administrateurs, une présentation détaillée. Les procès-verbaux de l'administration départementale du Nord concentrent les références laconiques. Évoquant la journée du 10 août 1793, les administrateurs indiquent qu'après la cérémonie officielle, « la journée est remplie de chants et de danses patriotiques » sans plus de précision. Quelques jours plus tard, le 12 septembre 1793, la présentation 


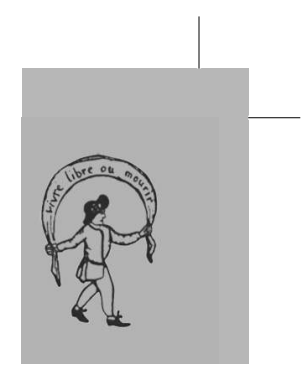

des jeunes de 18 à 25 ans recrutés pour former un nouveau corps dans l'armée donne lieu à des manifestations, et les administrateurs décrivent, de manière satisfaite, «l'air qui retentissoit de chansons patriotiques $»^{25}$.

Les témoignages sur les rassemblements collectifs que sont les fêtes, les assemblées électorales et les mouvements séditieux offrent des indications précieuses sur les modes d'expression collective des diverses tendances politiques. L'étude des célébrations officielles confirme des évolutions bien connues à l'échelle nationale. La comparaison de la fête organisée par les autorités départementales, à Douai, le 15 septembre 1791, avec celle en l'honneur de l'acte constitutionnel illustre le transfert de sacralité étudié par Mona Ozouf ${ }^{26}$. Lors de la première célébration un « Te Deum », et le « Domine salvam fic Regem » sont interprétés en l'église paroissiale de Saint Pierre; lors de la seconde cérémonie, un cortège avec trompettes et tambours défile dans les rues en entonnant «L'hymne de la Liberté $\gg{ }^{27}$. Toutefois une analyse fine des modalités d'interprétation lors des rassemblements officiels souligne la gageure qui consisterait à circonscrire une sphère autonome d'usages officiels. Cette difficulté s'explique par le double statut qu'endosse la population, à la fois public et interprète. La distinction nette entre manifestation encadrée et emploi spontané, entre pratiques officielles et expression populaire s'avère, à certains égards, artificielle. Le déroulement de la réunion de l'assemblée électorale du département du Nord, le 2 septembre 1792, dans l'église paroissiale du Quesnoy en offre l'illustration. L'élection du premier député, Merlin de Douai, s'accompagne d'une interprétation par les membres de l'assemblée du fameux « Ça ira ». Avons-nous affaire à une célébration officielle ou à une interprétation spontanée? Il semble davantage pertinent de noter la spécificité locale. Le « Ça Ira » dont les premières traces dans le département du Nord remontent à juillet 1790, date à laquelle les paroles sont publiées dans le journal lillois L'Abeille patriote, demeure une chanson emblématique aux yeux des électeurs alors que se diffusent avec succès, à la même période, des airs nouveaux comme « La Carmagnole ». Pour les électeurs, l'interprétation du « Ça Ira » concrétise une volonté d'afficher une unité et un optimisme prononcé dans un contexte périlleux, puisque les armées ennemies sont situées à quelques kilomètres du Quesnoy.

«La Marseillaise », à cette même date, ne suscite pas un enthousiasme massif dans le département du Nord. Les interprétations répétées

(25) Ibid., L 108.

(26) Mona Ozouf, La fête révolutionnaire, Paris, Gallimard, 1988 ( $1^{\mathrm{ère}}$ éd. 1976).

(27) AD Nord, L 111 
de « la Marseillaise» dans ce département sont, en fait, plus tardives. Elles révèlent également les effets de sens provoqués par cette imbrication de pratiques officielles et d'interprétations spontanées. Sans revenir sur les circonstances précises de son succès en France, relevons seulement que «la Marseillaise », composée à Strasbourg, en avril 1792, a notamment été popularisée à Paris durant l'été 1792 par les bataillons de volontaires en provenance de Marseille. Les récits de la diffusion de cette chanson suggèrent souvent une adhésion immédiate et enthousiaste. Mais dans le département du Nord, le grand moment de diffusion et de popularisation de la chanson est postérieur à l'été : il date de novembre 1792. En effet, conformément au décret de la Convention du 28 septembre 1792 qui ordonne de célébrer les victoires de l'armée française en Savoie, "la Marseillaise » est interprétée sur la place publique des villes et villages du département. Or cette diffusion par voie officielle a des implications concrètes à long terme, car elle déconnecte en partie la chanson de l'idée d'un élan patriotique spontané, pour l'articuler plus spécifiquement au succès d'un régime particulier : la République, durant son moment jacobin. En conséquence, alors qu'après le 9 Thermidor, puis la mise en place de la république directoriale, " la Marseillaise » demeure une référence symbolique assumée par le nouveau régime (elle devient même en messidor an III, hymne national), une population locale hostile aux jacobins exprime, à de nombreuses reprises, son refus de cet hymne, lui préférant le «Réveil du peuple », œuvre ouvertement anti-jacobine. Ainsi le 10 thermidor an VII, alors que les tensions s'exacerbent dans la région lilloise, « la Marseillaise est huée » et des cris « à bas les jacobins " sont lancés ${ }^{28}$. Les modalités initiales de diffusion de l'hymne ont donc contribué à l'associer à un mouvement politique et à un régime. Comme l'a justement remarqué Michel Vovelle, la composition chantée à Strasbourg en avril 1792, par ces modalités de circulation, va, en un sens, échapper à son auteur, pour être « appropriée et répercutée en écho jusqu'à devenir la Marseillaise $»^{29}$. En effet, Rouget de l'Isle, au moment de la composition de son œuvre en avril 1792, était, comme la majorité des officiers de l'armée française, royaliste. Il le demeurera malgré la suspension du pouvoir exécutif, comme l'indique, après le 10 août, son refus d'admettre les évolutions en cours qui provoque son départ de l'armée ${ }^{30}$.

(28) AD Nord, L 1242.

(29) Michel Vovelle «La Marseillaise : la guerre ou la paix », dans Lieux de mémoire, tome 2, «La Nation », Paris, Gallimard, 1986, p. 107-152.

(30) Marcel Reinhard, La chute de la royauté : 10 août 1792, Paris, Gallimard, 1969. 


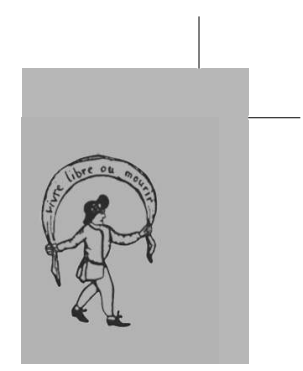

Le contexte de réception et d'interprétation a donc progressivement et profondément infléchi la portée emblématique de l'hymne.

L'adaptation spontanée ou du moins non encadrée de ces emblèmes nationaux aux conflits locaux participe également à des détournements de sens. Les œuvres d'origine locale sont rares en comparaison de la production parisienne, mais elles demeurent particulièrement significatives de l'articulation qui s'établit entre emblèmes nationaux et conflits locaux. Cette articulation se concrétise dans les modalités de production des chansons. Dans le département du Nord, toutes les chansons locales sont ainsi écrites sur des timbres nationaux, sur des airs à succès connus dans tout le pays. La question religieuse, sujet d'affrontements au sein des communautés villageoises du département, suscite, dès décembre 1791, plusieurs compositions originales. Le 2 décembre 1791, un grand nombre de villageois de Flines se regroupent sur la place face à l'Église, puis « criant comme ils font très fréquemment vive la calotte, vive les aristocrates; après avoir été un certain espace de temps à boire et à chanter la chanson de cent onze couplets qu'ils ont composée sur le curé constitutionnel et les démocrates de l'endroit, ils sortirent des cabarets proches de l'église où ils étoient assemblés, prirent des cocardes blanches aux chapeaux aiant à leur tête deux militaires ». Le curé constitutionnel est insulté et l'" on se mit à chanter la chanson aux 111 couplets jusque bien avancé dans la nuit $\rangle^{31}$. Malheureusement, aucune trace, à notre connaissance, ne nous est parvenue de cette composition, mais deux enseignements peuvent être dégagés. Tout d'abord, l'interprétation à portée politique de chansons n'est pas une spécificité urbaine. Ces œuvres tiennent, par ailleurs, une place primordiale dans la mise en scène des revendications collectives et contribuent à l'identification d'un groupe d'individus à une cause commune.

En juin 1793, les officiers municipaux d'Esne, village situé dans le district de Cambrai, se rendent chez Wibail, fermier de la commune âgé de 80 ans, et retrouvent une chanson, de composition locale, favorable au clergé réfractaire. Lors d'un interrogatoire, Wibail affirme que sa fille a retrouvé cette chanson devant sa maison, et dit ne pas savoir si cette chanson a été chantée chez lui. Pourtant Wibail entretient des relations étroites avec d'autres personnes témoignant d'une hostilité manifeste à l'égard du clergé constitutionnel : il reconnaît son amitié avec Delbart, lui aussi fermier octogénaire, chez qui sont retrouvés un Bref du Pape Pie VI et 
une « Déclaration de son altesse sérénissime le duc régnant de Brunswick et de Luxembourg ». Il affirme également être proche d'un dénommé Thuliez qui refuse de se rendre à la messe des prêtres assermentés. Si rien ne prouve que cette œuvre a été rédigée par l'un de ces trois protagonistes, elle n'en demeure pas moins caractéristique de leurs convictions.

Air : c'est ce qui me console, c'est ce qui me désole ${ }^{32}$

Deux prélats viennent en ce lieu, Se disant envoyé de Dieu

Voilà la ressemblance!

Par la douceur l'un nous soumet

L'autre s'énonce en Mahomet

Voilà la différence !

Rohan est notre vrai pasteur

Et Primat en a la couleur :

Voilà la ressemblance !

Des apôtres vient le premier,

Du côté gauche le dernier

Voilà la différence !

Tous deux sont crossés et mitrés

Tous les deux ont été sacrés :

Voilà la ressemblance!

L'un le fut par les vrais prélats,

L'autre par les apostats :

Voilà la différence !

Tous deux désirent nous gagner

Et sur nos cœurs veulent régner :

Voilà la ressemblance!

Rohan a les honnêtes gens

Et pour Primat sont les brigands :

Voilà la différence!

(32) AD Nord, L 859. 
Rohan donne des mandements

Et Jean Primat des documents :

Voilà la ressemblance!

L'un suit les pontifes romains

Et l'autre Luther et Calvin :

Voilà la différence!

Ils sont tous deux secondés

Par des vicaires et des curés :

Voilà la ressemblance!

Rohan donnera l'esprit sain

Et Jean Primat, l'esprit malin :

Voilà la différence!

Aux deux prélats, l'empressement

Procure un juste traitement

Voilà la ressemblance!

À l'un la grâce et la vertu

À l'autre CUPIDON BALENS

Voilà la différence!

L'Église et l'Europe ont jugé

Sur tous deux ont décidé

Voilà la ressemblance!

Que l'un au ciel nous conduira

Et l'autre au diable nous mènera

Voilà la différence!

Cette chanson est composée sur l'un des timbres les plus utilisés, durant la décennie révolutionnaire, parmi ceux qui sont issus du répertoire d'Ancien Régime. L'air intitulé « On doit soixante mille francs » ou «C'est ce qui me console, c'est ce qui me désole » provient de l'opéracomique Les Dettes, composé en 1787 par Champein; ce timbre est mentionné à 49 reprises dans le catalogue de Constant Pierre. Son succès, comme l'atteste l'adaptation mentionnée, n'est pas circonscrit à la sphère des auteurs parisiens. L'attrait de l'œuvre vient probablement de la structure binaire de chaque couplet fondé sur la répétition de jugements antagonistes « c'est ce qui me console [...] c'est ce qui me désole » dans le texte d'origine, "voilà la ressemblance [...] voilà la différence » dans l'adaptation septentrionale. Cette structure s'avère particulièrement pro- 
pice à la mise en valeur de discours édifiants qui visent à lever les fauxsemblants, les apparences trompeuses. L'exemple proposé est, en ce sens, significatif et s'inscrit pleinement dans la continuité des usages répétés de ce timbre. Mais, cette fois, les jugements antagonistes se portent sur deux personnages, incarnations locales des conflits religieux provoqués par la Constitution civile du clergé : Rohan ci-devant évêque de Cambrai, et Jean Primat, évêque constitutionnel du département du Nord.

L'action des villageois de Flines, comme la composition sur les deux prélats, suggère évidemment la permanence des pratiques de défiance voire de contestations motivées par la question religieuse dans les communautés villageoises. Ils révèlent surtout deux usages différents. Dans le premier cas, la chanson emblème d'un engagement est intégrée à une mise en scène des revendications collectives dans l'espace public. Dans le second cas, la chanson reste cantonnée à un usage privé et semble circuler à l'intérieur d'un réseau d'individus proches, partageant les mêmes convictions : elle entretient une dimension emblématique voire identitaire pour ce groupe d'individus hostiles au clergé assermenté, mais elle n'est plus exhibée dans un contexte nouveau où la répression des autorités devient plus menaçante. L'imbrication du contexte national et des configurations sociopolitiques locales joue donc un rôle prépondérant dans le processus de production et d'utilisation des œuvres.

L'espace flamand, par la virulence des conflits politiques locaux qui s'y déploient, par une spécificité culturelle claire marquée dans le recours à un dialecte spécifique, constitue un lieu d'étude privilégié pour saisir l'emboîtement des références symboliques. L'interprétation de chansons parodiques hostiles à la Révolution s'accompagne parfois de violences physiques, tout en polarisant les conflits locaux autour d'emblèmes nationaux. À Cassel en 1792, des cavaliers du «ci-devant régiment royal cravate » forcent au cabaret, après lui avoir donné quelques coups, un soldat volontaire à se mettre à genoux et à chanter des " chants contrerévolutionnaires $»$. Parmi ces chants, une composition attirera spécifiquement l'attention des administrateurs du district, il s'agit d'une parodie $\mathrm{du}$ « Ça ira». Cette fois, le refrain se caractérise par l'expression « les patriotes à la lanterne $»$. Les administrateurs du district indiquent que cette chanson présente des références nominales aux patriotes habitant la ville de Cassel $^{33}$. La pratique de la parodie locale n'est pas nouvelle, mais elle est ici réinvestie dans un contexte de tensions politiques fortes, dans une 
ville où les marques d'hostilité à la Révolution sont nombreuses. Cette pratique parodique qui perdure par-delà l'année 1792 n'est pas l'apanage d'individus hostiles à la Révolution française qui se réfugieraient dans un folklore ou dans des traditions locales. Dans un contexte de conflit local prolongé, les groupes antagonistes ont recours aux mêmes références musicales telles que le " Ça ira ", aux mêmes menaces envers des individus nommément cités, et au même dialecte. L'intensité des affrontements provoque donc des conflits d'usage, chaque groupe essayant de s'approprier les mélodies comme les procédés rhétoriques jugés les plus efficaces dans le but d'emporter un large soutien. En fructidor an III, à Hazebrouck, alors que le rapport de force local s'est, comme au niveau national, sensiblement modifié, les administrateurs du district s'inquiètent de rassemblements nocturnes, de la diffusion d'écrits et de l'interprétation de chansons dans les rues. Le 5 fructidor, des individus qualifiés de « jacobins désarmés » parcourent la ville en chantant une adaptation locale du « Ça Ira »: «Ah ça ira, paters kerke, on la fermera » ${ }^{34}$. L'interprétation publique s'inscrit dans un contexte de contestation locale marqué par des rassemblements mais aussi la diffusion de quatre pamphlets, dont les titres entrent en résonance avec l'adaptation du «Ça Ira » : le premier texte est intitulé « Les roalist ont punira, Le patriot triomphera, De Groote Klocke sonnera », le troisième « De Groote Klocke sonnera, Les terrorist vivera, Paters Kercke o diable ». Les mêmes formules, et le recours similaire à des menaces vis-à-vis d'individus clairement identifiés, indiquent que l'interprétation publique de la chanson, qui traduit un conflit d'usage entre groupes rivaux, s'intègre à un ensemble plus vaste de supports d'expression. La convergence de ces différents modes d'expression permet alors de saisir la nature et l'ampleur des oppositions locales.

En affinant l'analyse des conditions d'interprétations collectives à partir des exemples de Flines, de Cassel et d'Hazebrouck, un lieu mentionné dans chaque configuration retient l'attention, le cabaret. Les références témoignant de la place primordiale tenue par ces débits de boisson où se concrétise l'articulation entre pratique du chant et engagements collectifs, abondent. À Merville en prairial an V, les administrateurs du district constatent une nouvelle fois que «le parti le plus fort provoque le plus faible dans les cabarets par des propos et des chansons contre 
la constitution $»^{35}$. À la fois lieu de sociabilité et de divertissement, le cabaret tient notamment son attrait des chansons que l'on vient y interpréter ou écouter. Le rôle central des cabarets dans la cristallisation des mouvements de contestation n'est pas nouveau comme l'a souligné Jean Nicolas $^{36}:$ «Les rencontres de cabaret en un espace favorable au mixage social à humble niveau, facilitaient les prises de langue, le montage des coups irréguliers $»$. Lieu de fête et de cristallisation des mécontentements collectifs, le cabaret continue à occuper une place fondamentale à la ville comme au village durant la Révolution. L'analyse des pratiques témoigne donc à la fois de la persistance, dans la longue durée, des lieux et des supports d'expression de la contestation, tout en révélant des compositions originales associant emblèmes nationaux et références locales.

En refusant de restreindre l'approche des chansons au commentaire de texte, de nouveaux champs d'investigation s'ouvrent : ils portent aussi bien sur les liens qui se tissent entre différents espaces que sur les étapes de la cristallisation des opinions collectives. Les œuvres et leurs modalités d'interprétation constituent donc un objet d'étude transversal qui tient à la fois des configurations sociales, des luttes politiques et des pratiques culturelles. La chanson constitue un support dynamique parce que ce support s'inscrit dans des stratégies de circulation complexes, et parce que, in fine, cette circulation contribue à faire de ce support un mode d'expression propice aux détournements de sens comme l'a souligné Jean Quéniart ${ }^{37}$.

Par conséquent, si l'analyse formelle des chansons semble témoigner du partage par différents partis des mêmes références symboliques et mélodiques, l'étude des pratiques nous montre que ce constat, loin d'être l'illustration d'une culture uniforme voire consensuelle, relève, en fait, de conflits d'usage intenses qui caractérisent les luttes politiques à l'échelle locale comme nationale. Chaque parti tente de s'approprier les mélodies et les symboles « dans l'air du temps » afin d'accroître sa légitimité et d'emporter l'adhésion du plus grand nombre.

(35) AD Nord L 880.

(36) Jean Nicolas La rébellion française, mouvements populaires et conscience sociale, 1661-1789, Paris, Seuil, 2002.

(37) Jean QuÉNIART «Conclusion », dans J. QuÉNIART (dir.), Le chant acteur de l'histoire, Actes du colloque de Rennes du 9 au 11 septembre 1998, Rennes, Presses Universitaires de Rennes, 1999 , p. 347-356. 
Toutefois, les emplois spontanés, les parodies constantes enrayent les velléités de promotion d'une propagande strictement encadrée. L'interprétation des œuvres encourage et exprime, avant tout, une participation politique élargie mais difficile à orienter de manière décisive. La pratique de la chanson est donc, tout à la fois, « un symptôme et un agent», pour reprendre l'expression de François Ploux ${ }^{38}$. Elle agit puisqu'elle participe au développement local de discours et d'actions collectives dans le domaine public. Elle constitue un symptôme car ses interprétations s'ancrent profondément dans un contexte particulier où s'affirment des craintes et des espérances spécifiques.

Maxime KACI

Doctorant

IRHIS - Université Charles-de-Gaulle-Lille3 UFR Sciences historiques artistiques et politiques BP 60149 59653 Villeneuve d'Ascq Cedex maxime.kaci@gmail.com 